\title{
Plaque Inflammation Imaging in Severe Carotid Stenosis and Recurrent Cerebral Ischemia
}

\author{
Vijay K. Sharma ${ }^{1,2}$, Prakash R. Paliwal ${ }^{1}$, and Arvind K. Sinha ${ }^{3}$ \\ ${ }^{1}$ Division of Neurology, National University Hospital, Singapore; ${ }^{2} Y L L$ School of Medicine, National University of Singapore, \\ Singapore; and ${ }^{3}$ Department of Diagnostic Imaging, National University Hospital, Singapore
}

A strong relationship exists between the severity of carotid stenosis and early stroke risk. Inflammation is believed to be an important event for atherosclerotic plaque destabilization and subsequent thromboembolism. ${ }^{18} \mathrm{~F}-\mathrm{FDG}$ can image atherosclerotic inflammation, providing information about plaque biology, which may serve as a useful biomarker for the assessment of early stroke risk.

Key Words: acute ischemic stroke; carotid stenosis; plaque inflammation; PET/CT

J Nucl Med Technol 2015; 43:299-300

DOI: 10.2967/jnmt.114.153627

\section{A}

strong relationship exists between the severity of carotid stenosis and early stroke risk that is independent of other prognostic indicators and neuroimaging findings $(1,2)$. Inflammation may lead to atherosclerotic plaque destabilization and thromboembolism (3). Increased ${ }^{18}$ F-FDG uptake represents carotid plaque inflammation on PET and predicts early recurrent stroke, independent of age and degree of stenosis (4).

\section{CASE REPORT}

A 58-y-old man presented with transient right hemiparesis and aphasia. His cardiovascular risk factors included hypertension, ischemic heart disease, and dyslipidemia. He had been diagnosed $2 \mathrm{y}$ previously with asymptomatic severe $(>70 \%)$ stenosis of the left internal carotid artery. The findings on clinical examination and CT scanning of the brain were unremarkable. CT angiography (Fig. 1A) confirmed the preexisting severe focal stenosis of the left internal carotid artery.

Clopidogrel, aspirin, and atorvastatin were started with a plan for early carotid endarterectomy. However, on day 3 he developed right hemiparesis. Brain MR imaging revealed multiple acute ischemic infarctions in the left middle and

Received Dec. 29, 2014; revision accepted Feb. 4, 2015.

For correspondence or reprints contact: Vijay K. Sharma, Division of Neurology, National University Health System, Level 10, Tower Block, 1E Kent Ridge Rd., Singapore 119228.

E-mail: drvijay@singnet.com.sg

Published online Apr. 9, 2015.

COPYRIGHT (C) 2015 by the Society of Nuclear Medicine and Molecular Imaging, Inc. anterior cerebral artery territories (Fig. 1B). Transcranial Doppler monitoring of the left middle cerebral artery revealed spontaneous microemboli (Fig. 1C). Carotid PET/CT fused with CT angiography showed increased ${ }^{18} \mathrm{~F}-\mathrm{FDG}$ uptake in the left carotid plaque (Figs. 2A and 2B), representing acute inflammation. His family members refused an urgent carotid endarterectomy. On day 5, he developed another severe ischemic stroke in the left middle cerebral artery, with clinical worsening. His further stay in the hospital was uneventful until discharge to a rehabilitation unit.

\section{DISCUSSION}

Our case demonstrates the role of ${ }^{18} \mathrm{~F}$-FDG PET imaging of carotid plaque inflammation as a reliable marker for risk stratification. To date, risk stratification for carotid atherosclerosis has been based mainly on arterial lumen stenosis, and understanding of plaque biology remains poor (1).

Limited data are available on the prognostic utility of metabolic imaging with PET to predict short-term stroke risk in patients with recently symptomatic carotid atherosclerosis. The addition of plaque ${ }^{18} \mathrm{~F}-\mathrm{FDG}$ data to the Framingham Risk Score significantly improved long-term risk prediction (5). In a study in Ireland, the predictive utility of ${ }^{18} \mathrm{~F}-\mathrm{FDG}$ plaque uptake was independent of age and degree of lumen

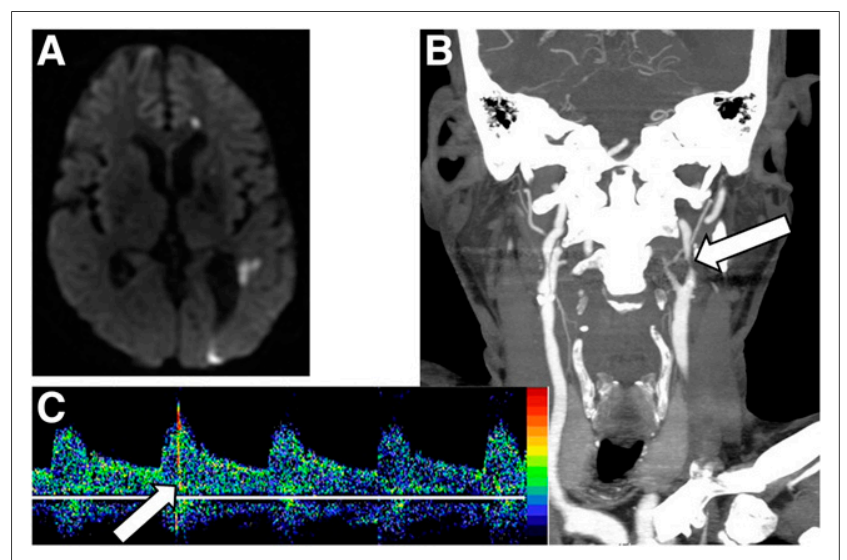

FIGURE 1. Diffusion-weighted MR imaging (A) shows ischemic infarcts in left internal carotid artery territory with highgrade stenosis (arrow) on CT angiography (B). Transcranial Doppler monitoring shows spontaneous microembolic signals (arrow) (C). 

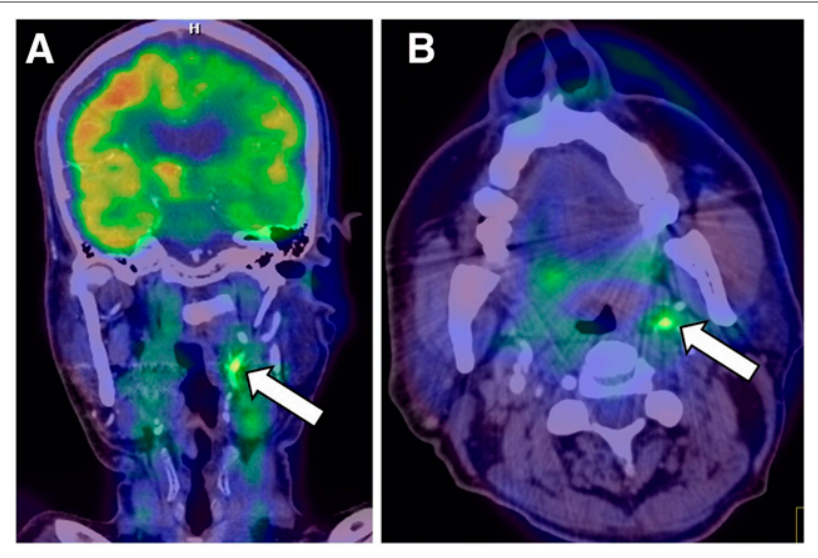

FIGURE 2. PET/CT imaging shows increased uptake of ${ }^{18}$ F-FDG (arrows) in left internal carotid artery plaque on coronal plane (A) and axial plane (B), suggestive of acute inflammation.

stenosis (categorized as 50\%-69\% and 70\%-99\%; adjusted hazard ratio, 6.1; confidence interval, 1.3-28.8; $P=0.02$ ) (4). In addition to risk stratification, molecular imaging with ${ }^{18}$ F-FDG PET may have potential to monitor the response of atherosclerotic plaque to therapeutic intervention.

\section{CONCLUSION}

Inflammation in a previously stable carotid plaque is an important event that leads to local thrombosis and cerebral embolization. This phenomenon can be reliably imaged with ${ }^{18} \mathrm{~F}$-FDG PET for better risk stratification and therapeutic strategizing in carotid stenosis.

\section{DISCLOSURE}

No potential conflict of interest relevant to this article was reported.

\section{REFERENCES}

1. Sheehan OC, Kyne L, Kelly LA, et al. A population based comparison of ABCD2 score, atrial fibrillation and carotid stenosis for prediction of early stroke recurrence after transient ischaemic attack. The North Dublin TIA Study. Stroke. 2010;41:844-850.

2. Merwick A, Albers GW, Amarenco P, et al. Addition of brain and carotid imaging to the $\mathrm{ABCD}^{2}$ score to improve identification of patients at high early stroke risk after transient ischaemic attack. Lancet Neurol. 2010;9:1060-1069.

3. Libby P, Ridker P, Hansson GK. Progress and challenges in translating the biology of atherosclerosis. Nature. 2011;473:317-325.

4. Marnane M, Merwick A, Sheehan OC, et al. Carotid plaque inflammation on ${ }^{18}$ FDG-PET predicts early stroke recurrence: the Dublin Carotid Atherosclerosis Stroke Study. Ann Neurol. 2012;71:709-718.

5. Tawakol A, Finn AV. Imaging inflammatory changes in atherosclerosis multimodal imaging hitting stride. JACC Cardiovasc Imaging. 2011;4:1119-1122. 